\title{
Erratum to: Micro real-time PCR device using a circulation pump
}

\author{
Byung-Phil Mun ${ }^{1}$ Chang-Ju Park ${ }^{2} \cdot$ Jae-Kwon Lee $^{1} \cdot$ Kyoungsook Park $^{3}$ \\ Jong-Hyun Lee ${ }^{1,2}$
}

Published online: 14 July 2017

(C) Springer-Verlag GmbH Germany 2017

\section{Erratum to: Microsyst Technol \\ DOI 10.1007/s00542-016-3031-z}

Unfortunately, author names were included twice in the original online publication of this article.

The correct author names are given here. The original article was corrected.

Byung-Phil Mun, Chang-Ju Park, Jae-Kwon Lee, Kyoungsook Park, Jong-Hyun Lee

The online version of the original article can be found under doi:10.1007/s00542-016-3031-z.

Jong-Hyun Lee

jonghyun@gist.ac.kr

1 School of Mechatronics, Gwangju Institute of Science and Technology (GIST), 123 Cheomdann-gwagiro, Buk-gu, Gwangju 500-712, Republic of Korea

2 Department of Medical System Engineering, Gwangju Institute of Science and Technology (GIST), 123 Cheomdann-gwagiro, Buk-gu, Gwangju 500-712, Republic of Korea

3 BioNano Health Guard Research Center, Korea Research Institute of Bioscience and Biotechnology (KRIBB), 125 Gwahangno, Yuseong, Daejeon 305-806, Republic of Korea 